Eur. J. Clin. Chem. Clin. Biochem.

Vol. 32, 1994, pp. 145-152

(c) 1994 Walter de Gruyter \& Co. Berlin - New York

\title{
Harnstoff-Durchflußsensor zur ammoniumselektiv-enzymatischen Prozeßkontrolle der Künstlichen Niere
}

\author{
Von J. G. Schindler ${ }^{1}$, M. M. Schindler ${ }^{2}$, K. Herna ${ }^{1}$, E. Reisinger ${ }^{1}$, B. Burk ${ }^{1}$, U. Kuhlmann ${ }^{3}, J$. Knaack $^{3}$, \\ B. Schmidt ${ }^{3}$ und $H$. Lange ${ }^{3}$ \\ I Institut für Normale und Pathologische Physiologie, Projekt Biomedizinische Technik und Bioelektrochemische \\ Membranelektroden, Philipps-Universität, Marburg/Lahn, Germany \\ 2 Schindler Bio- und Chemosensoren Forschungslabor, Marburg/Lahn \\ ${ }^{3}$ Medizinisches Zentrum für Innere Medizin, Abteilung Nephrologie, Philipps-Universität, Marburg/Lahn
}

(Eingegangen am 18. Oktober 1993/3. Januar 1994)

Zusammenfassung: Es wird ein Durchfluß-Verfahren zur kontinuierlichen ammoniumselektiv-enzymatischen Prozeßkontrolle der Künstlichen Niere mit einer bioelektrochemischen Harnstoff-Elektrode vorgestellt. Die Harnstoffspaltung erfolgt mittels membranständiger kovalent gebundener Urease, die Detektion der freigesetzten Ammoniumionen durch eine Nonactin-PVC-Membran. Außer detaillierten Kenndaten des sauerstoffunabhängigen Festkontaktsensors werden patientendirekte kontinuierlich gewonnene Meßkurven während des Hämodialyseverfahrens demonstriert. Verfahrenstechnische Vorteile sind ausführlich beschrieben. Darüberhinaus gestattet der Harnstoff-Sensor auch Direktmessungen im heparinisierten Blut.

Urea-sensor for the continuous ammonium-selective-enzymatic process control of the artificial kidney

Summary: Presented is a flow-through method for continuous ammonium-selective enzymatic monitoring of the artificial kidney by means of a bioelectrochemical urea electrode. The urea is converted in an enzyme membrane by covalently bound urease and the ammonium ions are detected by a Nonactin-PVC-membrane. In addition to detailed data from the oxygen-independent solid-state contact sensor, curves are obtained on-line from the patient during the haemodialysis session. The advantages of the method are described in detail. Furthermore, the urea sensor can be used for measurements in heparinized blood.

\section{Einführung}

Die kontinuierliche Meßwerterfassung von $\mathrm{K}^{+}, \mathrm{Na}^{+}$und $\mathrm{Ca}^{2+}$ bei chronisch nièreninsuffizienten Patienten während der Hämodialyse haben wir in Marburg 1987/88 eingeführt und verschiedentlich darüber publiziert $(1-3)$. Da das realzeitliche On-Line-Harnstoff-Monitoring als zukünftiger idealer Weg zur Erzielung gleicher Effektivität dieser Behandlungsform angesehen wird (4-6), wurde von uns die Elektroanalyse der Kationen durch einen Harnstoff-Biosensor (7) erweitert. In diesem Zusammenhang haben wir kürzlich über die fortlaufende Harnstoff-Messung im abfließenden Dialysat an der Künstlichen Niere berichtet (8).

Als zukünftige Perspektive eröffnet sich für den Arzt die Möglichkeit, den Eiweißmetabolismus des Patienten über eine Bestimmung der exkorporierten Harnstoffmenge besser beurteilen zu können. Darüberhinaus gestattet die dafür erforderliche gleichzeitige kontinuierliche Bestimmung des Harnstoffs im Filtrat des Blutes und im abfließenden Dialysat eine Berechnung der Harnstoffclearance der Künstlichen Niere. Schließlich leistet das Verfahren auch noch einen Beitrag zur Qualitätssicherung des hämodialytischen Behandlungsverfahrens.

\section{Material und Methodik}

Das bioelektrochemische Meßprinzip (7) der Harnstoff-Elektrode (Abb. 1) basiert auf der enzymatischen Spaltung durch membran- 


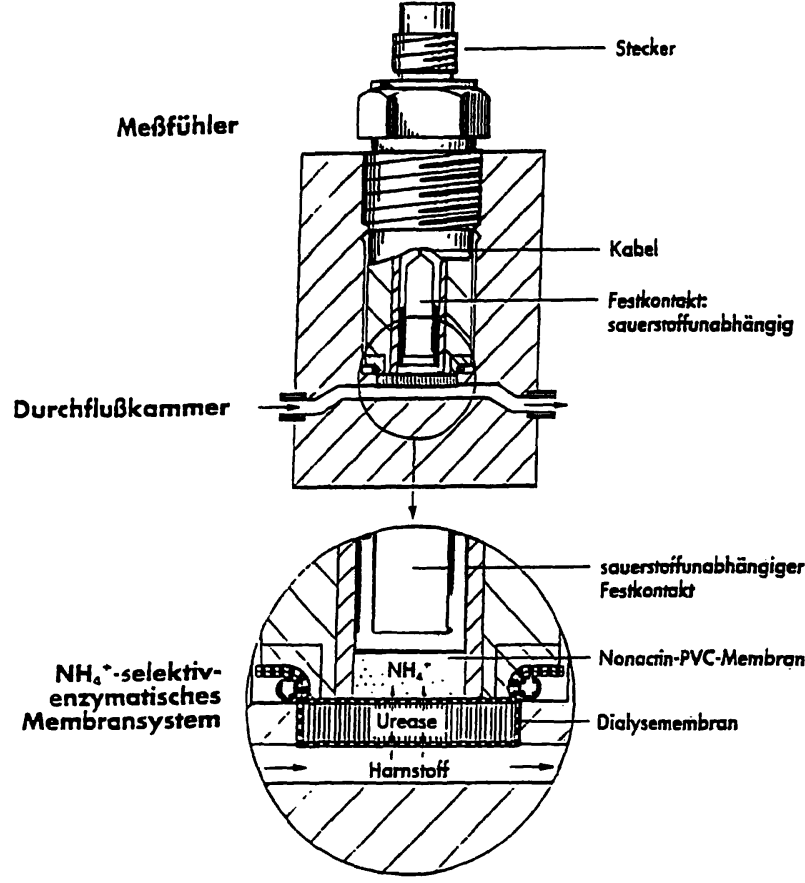

Reaktionsigleichung: Harnstoff $+2 \mathrm{H}_{2} \mathrm{O}+\mathrm{H}^{+} \stackrel{\text { Ureaso }}{\longrightarrow} 2 \mathrm{NH}_{4}^{+}+\mathrm{HCO}_{3}^{-}$

Abb. 1 Bioelektrochemische Harnstoff-Membranelektrode.

ständige kovalent gebundene Urease und elektrochemischer Detektion der freigesetzten Ammoniumionen hinter der Enzymmembran durch eine Nonactin-Disk-Elektrode $(7,9)$ mit sauerstoffunabhängiger Festableitung gemäß der Reaktionsgleichung

$$
\text { Harnstoff }+2 \mathrm{H}_{2} \mathrm{O}+\mathrm{H}^{+} \stackrel{\text { Urease }}{\longrightarrow} 2 \mathrm{NH}_{4}^{+}+\mathrm{HCO}_{3}^{-}
$$

Die Urease-Membran aus quervernetzten Enzymmolekülen ist beidseits durch Dialysemembranen versteift und in dieser mechanisch stabilisierten Form durch einen O-Ring auf einem Membranträger aus Acrylglas arretiert, der auf die Nonactin-Disk-Elektrode aufgeschoben wird; dadurch läßt sich bei nachlassender Aktivität die Enzymmembran leicht auswechseln. Bis zu ihrem Einsatz ist eine Lagerung über mehrere Monate bei $+4{ }^{\circ} \mathrm{C}$ im Kühlschrank möglich.

Die aus Harnstoff enzymatisch freigesetzten Ammoniumionen stehen einerseits in der Meßlösung pH-abhängig mit $\mathrm{NH}_{3}$ im Gleichgewicht (9) und werden andererseits unter Abstreifen der Hydrathülle im Zuge einer Phasentransferkatalyse durch das in die PVC-Membran inkorporierte exolipophile Makrotetrolid-Antibiotikum Nonactin mit je vier Carbonyl- und Tetrahydrofuransauerstoffatomen achtfach in geringfugig verzerrter kubischer Koordinationssphäre $(10)$ komplexiert. Die $\mathrm{NH}_{4}^{+}$-Ionen finden in diesen $\mathrm{Li}$ gandsauerstoffatomen einen Ersatz für ihre Hydrathülle, so daß sie nun als lipophile Komplexe in der Membranphase vorliegen. Der Chemismus des Komplexierungsvorganges begründet auf der Basis einer Bildung ligandgetrennter Ionenpaarè die aktivitätsgetriebene Ausbildung der Potentialdifferenz im Grenzflächenbereich von Nonactin-PVC-Membran und hydrophiler Enzymmembran, wobei sich Ammoniumionen-Nonactin-Komplexe und hydratisierte Anionen im Sinne zweier Kondensatorplatten gegenüberstehen.

Feine Unterschiede in der räumlichen Ausdehnung der Koordinationssphäre des achtzähnigen Liganden lassen bei seinen $\mathrm{NH}_{4}^{+}$, $\mathrm{K}^{+}$- und $\mathrm{Na}^{+}$-Komplexen den Zusammenhang von Struktur und Selektivität erkennen. Während das nicht hydratisierte $\mathrm{K}^{+}$-Ion im endohydrophilen Hohlraum $8 \mathrm{fach}$ näherungsweise kubisch (ungefähr 2,8 $\times 10^{-10} \mathrm{~m}$ ) koordiniert vorliegt (11), nähern sich im Falle einer Natriumkomplexierung die Carbonylsauerstoffatome dem Metallkation auf eine Distanz von circa $2,4 \times 10^{-10} \mathrm{~m}(12)$, hinge- gen distanzieren sie sich beim Ammonium-Komplex vom Stickstoffatom auf eine Entfernung von $3,01-3,16 \times 10^{-10} \mathrm{~m}$ (10), so daß die Wasserstoffatome von $\mathrm{NH}_{4}^{+}$mit den Tetrahydrofuran-Sauerstoffatomen (ungefähr $2,0 \pm 0,15 \times 10^{-10} \mathrm{~m}$ ) in besondere Wechselwirkung treten. Der Abstand der Tetrahydrofuran-OAtome bleibt in den $\mathrm{K}^{+}$-, $\mathrm{Na}^{+}$- und $\mathrm{NH}_{4}^{+}-(\mathrm{N}$...O) Komplexen des Nonactins mit circa $2,8 \times 10^{-10} \mathrm{~m}$ ungefahr gleich $(10-12)$. Die exolipophile Peripherie des Molekül's'wird durch Methylengruppen der Tetrahydrofuranringe sowie Methylsubstituenten repräsentiert (11) und gewährleistet dadurch sein Verbleiben in der hydrophoben Membranphase.

Die Selektivitätsbestimmung des aus Nonactin, Bis(2-ethylhexyl)sebacat und PVC bestehenden Membransystems erfolgte gemäß IUPAC-Empfehlung (13) entsprechend der fixierten Interferenzmethode nach dem Prinzip der Veränderung der Meßionenaktivität bei konstant gehaltener Störionenkonzentration $(1,9)$. Die Ionenaktivitäten wurden auf der Basis der Debye-Hückel-Onsager-Theorie berechnet $(1,9)$. Da die Meßwerterfassung über Ammoniumionen Nernst-gemäßes Verhalten zeigt, kann für die bioelektrochemische Bestimmung des Harnstoffs formuliert werden:

$$
\begin{aligned}
\mathrm{E} & =\mathrm{E}^{\prime}+\mathrm{S} \lg c_{\text {Harnstoff }}[\mathrm{mV}] \\
\mathrm{E} & =\text { EMK der Meßkette } \\
\mathrm{E}^{\prime} & =\text { Standardmeßkettenpotential } \\
\mathrm{S} & =\text { Elektrodensteilheit } \\
c_{\text {Harnstoff }} & =\text { Konzentration des Harnstoffs in der Meßlösung }
\end{aligned}
$$

Für Durchflußanalysen wird die Harnstoff-Elektrode durch eine $\mathrm{Ag}$-Referenzelektrode mit $3 \mathrm{~mol} / \mathrm{l} \mathrm{KCl}$-Bezugslösung und monokapillarer Stromschlüsselkontaktzone (9) zur Meßkette mit Überfựhrung komplettiert.

Die Positionierung der beiden jeweils durch Tropfkammern vom Patienten abgetrennten bioelektrochemischen Harnstoff-Membranelektroden zur Prozeßkontrolle an der Künștlichen Niere geht aus Abbildung 2 hervor. Dabei dient der Sensor I der Ërfassung der aktuellen Blut-Harnstoffkonzentration des Patienten, die unter Anwendung einer entsprechenden Filtrationseinheit über eine fortlaufende Messung im Filtrat des Blutes registriert wird. Als wesentliche Funktion übernimmt der Sensor II im Zusammenhang mit einem Volumenmeßgerät die kontinuierliche Detektion der eliminierten Harnstoffmenge im abfließenden Dialysat. Über dieses Verfahren wird an anderer Stelle detailliert berichtet werden (14).

Die Kalibrierlösungen enthielten 40 und $100 \mathrm{mg} / \mathrm{dl}(6,67$ und 16,67 $\mathrm{mmol} / \mathrm{i})$ Harnstoff. Wegen der erheblich differierenden ionalen $\mathrm{Zu}$ sammensetzung von Filtrat und abfließendem Dialysat kommen auf der Basis von Phosphatpuffersystemen $\left(\mathrm{Na}_{2} \mathrm{HPO}_{4}\right.$ und $\left.\mathrm{KH}_{2} \mathrm{PO}_{4}\right)$ mit einem pH-Wert von 7,40 zwei unterschiedliche Kalibrierverfahren zur Anwendung (15). Die geringen Unterschiede in den Ionenradien von $\mathrm{NH}_{4}^{+}$und $\mathrm{K}^{+}$begründen eine vergleichsweise zu anderen Kationen in der Reihe der Alkali- und Erdalkalimetalle analytisch unerwünscht sehr hohe Querempfindlichkeit der Nonactin-PVC-Membran gegenüber diesem Störion. Diese und die Anio-

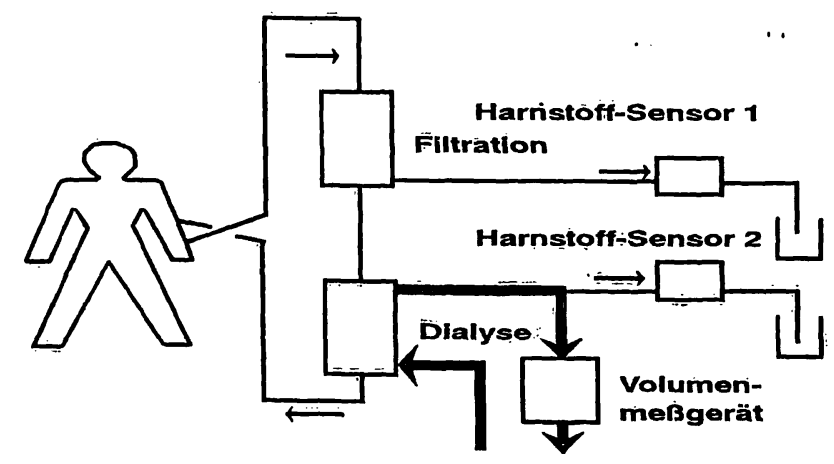

Abb. 2 Positionierung der Harnstoff-Sensoren zur Prozeßkontrolle an der Künstlichen Niere. ' 
neninterferenz verlangen eine an den Meßlosungen und am photometrischen Referenzverfahren orientierte Angleichung der Kalibrierlösungen bezüglich der Kalium- und Phosphatkonzentrationen. Daruberhinaus beeinflußt das Natriumion konzentrationsmäßig maßgeblich die Ionenstärke und übernimmt auf der $\mathrm{Ka}$ tionenseite die Funktion des Leitelektrolyten. Den verschiedenen Acetatkonzentrationen bei der sog. Bicarbonat- bzw. Acetatdialyse ist bei Messungen im abfließenden Dialysat gesondert Rechnung zu tragen.

\section{Ergebnisse}

Die Elektroanalytik des Harnstoffs ist zwischen 1 und $500 \mathrm{mg} / \mathrm{dl}(0,17-83,34 \mathrm{mmol} / \mathrm{l})$ ausführbar, jedoch werden auch Harnstoffkonzentrationen von $1000 \mathrm{mg} / \mathrm{dl}$ $(166,69 \mathrm{mmol} / \mathrm{l})$ erfaßt.

Die Gesamt-Selektivität der ammoniumselektiv-enzymatischen Harnstoffmembran gliedert sich in die Kationenselektivität der Nonactin-PVC-Membran und deren Anioneninterferenz sowie Konkurrenzreaktionen anderer Substrate mit Harnstoff um das aktive Zentrum der Urease bzw. deren Inhibition durch Hemmstoffe. Die nach der fixierten Interferenzmethode $(1,9,13)$ ermittelte Kationenselektivität geht aus Tabelle 1 hervor. $\mathrm{Da}$ der Unterschied des Ionenradius ( $\mathrm{r}_{\mathrm{lon}}$ nach Pauling) von $\mathrm{K}^{+}\left(\mathrm{r}_{\text {Ion }} 1,33 \times 10^{-10} \mathrm{~m}\right)$ gegenüber dem des $\mathrm{NH}_{4}^{+}\left(\mathrm{r}_{\text {Ion }}\right.$ $\left.1,48 \times 10^{-10} \mathrm{~m}\right)$ wesentlich geringer als bezüglich des $\mathrm{Na}^{+}\left(r_{\text {Ion }}\right.$ von $\left.0,95 \times 10^{-10} \mathrm{~m}\right)$ ist, wurden zur Quantifizierung der Kaliuminterferenz Harnstoffmessungen in Gegenwart anderer im Blut vorkommender Kationen wie $\mathrm{Na}^{+}(140,0 \mathrm{mmol} / \mathrm{l})$ und $\mathrm{Ca}^{2+}(1,17 \mathrm{mmol} / \mathrm{l})$ unter Variation der Kaliumkonzentrationen zwischen 3,00 und

Tab. 1 Characteristica der ammoniumselektiven Disk-Elektrode mit der Membran SBC-256- $\mathrm{NH}_{4}^{+}$bei $25^{\circ} \mathrm{C}$.

Selektivität auf der Basis dẹ fixierten Interferenzmethọde

\begin{tabular}{llll}
\hline $\begin{array}{l}\text { Störion } \\
\mathrm{S}^{\mathrm{n}}\end{array}$ & $\mathrm{K}_{\mathrm{NH}_{4}^{+}-\mathrm{S}^{\mathrm{n}+}}$ & $\begin{array}{l}\text { Störion } \\
\mathrm{S}^{n+}\end{array}$ & $\mathrm{K}_{\mathrm{NH}^{+}-\mathrm{S}^{\mathrm{n}+}}$ \\
\hline $\mathrm{H}^{+}$ & $3,67 \times 10^{-4 *}$ & $\mathrm{NH}_{4}^{+}$ & 1,00 \\
$\mathrm{Li}^{+}$ & $7,12 \times 10^{-5 *}$ & $\mathrm{Mg}^{2+}$ & $1,37 \times 10^{-5 *}$ \\
$\mathrm{Na}^{+}$ & $1,68 \times 10^{-3 *}$ & $\mathrm{Ca}^{2+}$ & $2,03 \times 10^{-5 *}$ \\
$\mathrm{~K}^{+}$ & $1,43 \times 10^{-10}$ & $\mathrm{Sr}^{2+}$ & $1,70 \times 10^{-5 *}$ \\
$\mathrm{Rb}^{+}$ & $6,03 \times 10^{-20}$ & $\mathrm{Ba}^{2+}$ & $8,79 \times 10^{-6 *}$ \\
$\mathrm{Cs}^{+}$ & $7,92 \times 10^{-30}$ & & \\
\hline
\end{tabular}

${ }^{*} c_{\mathrm{NH}}: 10^{-4}$ und $10^{-5} \mathrm{~mol} / \mathrm{l}, \mathrm{c}_{\mathrm{S}^{n+}}: 1,5 \times 10^{-1} \mathrm{~mol} / \mathrm{l}$ bzw. $\mathrm{c}_{\mathrm{H}^{+}}$: $10^{-1} \mathrm{~mol} / 1$

${ }^{-} c_{\mathrm{NH} t}: 10^{-3}$ und $10^{-4} \mathrm{~mol} / \mathrm{l}, \mathrm{c}_{\mathrm{S}^{\mathrm{n}}}: 10^{-2} \mathrm{~mol} / \mathrm{l}$

Elektrodensteilheit: $59,2 \mathrm{mV}$ pro Aktivitätsdekade

Einstellzeit: $<1 \mathrm{~s}$

Untere Nachweisgrenze (limit of detection): $1,5 \times 10^{-7} \mathrm{~mol} / \mathrm{l}$ $\mathrm{NH}_{4} \mathrm{Cl}$

Drift: $<0,1 \mathrm{mV} / \mathrm{h}$

Reproduzierbarkeit der EMK: 0,1 mV

Funktionsdauer: ca. 6 Monate
$7,00 \mathrm{mmol} / \mathrm{l}$ ausgeführt (Tab. 2). Der ionale Erkennungsmechanismus durch Nonactin erfolgt im Zuge der Phasentransferkatalyse nach dem Prinzip der optimalen Hohlraumanpassung. Aus der Sicht des Selektivitätsmechanismus erübrigt sich deshalb die Berücksichtigung $\operatorname{des} \mathrm{Mg}^{2+}\left(\mathrm{r}_{\mathrm{Ion}} 0,65 \times 10^{-10} \mathrm{~m}\right)$ in diesen Modelluntersuchungen, zumal bereits das Störion $\mathrm{Ca}^{2+}\left(\mathrm{r}_{\text {Ion }} 0,99\right.$ $\times 10^{-10} \mathrm{~m}$ ) nur noch einen vernachlässigbaren Beitrag zur Potentialbildung unter diesen Bedingungen leistet; denn hier wirkt sich bei diesen beiden Kationen noch die höhere Ladungszahl auf ihre Diskriminierung drastisch unterstützend aus.

Nach den Richtlinien der Bundesärztekammer zur Qualitätssicherung in medizinischen Laboratorien mit Gültigkeit vom 1. November 1988 (16) beträgt die jeweilige maximal zulässige relative zufällige Meßabweichung für Harnstoff $8 \%$. Im Hinblick darauf belegen die Ergebnisse in Tabelle 2, daß mit diesem Sensor elektroanalytische Harnstoffmessungen im Konzentrationsbereich von 30 bis $300 \mathrm{mg} / \mathrm{dl}(5,00$ bis $50,01 \mathrm{mmol} / \mathrm{l})$ bei einer Schwankung der Kaliumkonzentration zwischen 3,00 und 7,00 mmol/l keiner kaliumabhängigen Korrektur bedürfen. Da die Kalibrierlösungen bei diesen auf Tabelle 2 bezogenen Experimenten eine $\mathrm{K}^{+}$-Konzentration von $4,50 \mathrm{mmol} / \mathrm{l}$ aufwiesen, ist in Gegenwart von nur 30 $\mathrm{mg} / \mathrm{dl}$ (5,00 mmol/l) Harnstoff der Beitrag dieses Störions zur Potentialbildung bei steigender Kaliumkonzentration im Sinne einer positiven Meßwertabweichung und umgekehrt bei sinkender Kaliumkonzentration als negative Meßwertabweichung zu beobachten. Oberhalb $70,0 \mathrm{mg} / \mathrm{dl}(11,67 \mathrm{mmol} / \mathrm{l})$ Harnstoff sind die $\mathrm{zu}$ beobachtenden Meßwertabweichungen als zufällig einzustufen; denn nun vermögen die enzymatisch freigesetzten Ammoniumkonzentrationen die bei niedrigen Harnstoffkonzentrationen beobachteten Kaliuminterferenzerscheinungen vollständig zu kompensieren. Um generell Kaliumquerempfindlichkeiten drastisch zu minimieren, wurden die Kalibrierlösungen aber auch unter Berücksichtigung der Anioneninterferenz nach Maßgabe des oben beschriebenen Verfahrens modifiziert. Der Erfolg dieser Vorgehensweise wird in Abbildung 3 bestätigt.

Da einerseits zwischen $\mathrm{NH}_{4}^{+}$-Ionen und $\mathrm{NH}_{3}$ eine $\mathrm{pH}-$ abhängige Gleichgewichtseinstellung existiert und andererseits am enzymatischen Harnstoffumsatz durch die Urease Protonen beteiligt sind, wurde die $\mathrm{H}^{+}$-Interferenz des Sensors ebenfalls im Harnstoff-Konzentrationsbereich von 30 bis $300 \mathrm{mg} / \mathrm{dl}$ (5,00 bis $50,01 \mathrm{mmol} / \mathrm{l})$ unter Variation des $\mathrm{pH}$-Wertes zwischen 7,10 und 7,70 geprüft und auch als nicht korrekturbedürftig befunden (Tab. 3). Von der Nonactin-PVC-Membran ließ der Selektivitätskoeffizient $\mathrm{K}_{\mathrm{NH} \ddagger-\mathrm{H}^{+}}$(Tab. 1) ohnehin keine Protoneninterferenzen erwarten. 
Tab. 2 Kaliuminterferenz des Harnstoff-Sensors auf der Basis der Nonactin-PVC. Membran. Angegeben sind die vorgegebenen Harnstofkonzentrationen (..Waage"), die mit dem Sensor gemessenen
Harnstoffkonzentrationen $(\mathrm{mg} / \mathrm{dl})$ und die Abweichungen gegenüber den vorgegebenen $(\Delta, \%)$ sowie die korrespondierenden Konzentrationen in $\mathrm{mmol} / \mathrm{l}$.

\begin{tabular}{|c|c|c|c|c|c|c|c|c|c|c|}
\hline \multirow{2}{*}{\multicolumn{2}{|c|}{ Harnstoff, Waage }} & \multicolumn{9}{|l|}{$\mathrm{K}^{+}$} \\
\hline & & \multicolumn{3}{|c|}{$\begin{array}{l}3,00 \mathrm{mmol} / \mathrm{l} \\
\text { Harnstoff, Sensor }\end{array}$} & \multicolumn{3}{|c|}{$\begin{array}{l}4,50 \mathrm{mmol} / 1 \\
\text { Harnstoff, Sensor }\end{array}$} & \multicolumn{3}{|c|}{$\begin{array}{l}7,00 \mathrm{mmol} / \mathrm{l} \\
\text { Halmstoff, Sensor }\end{array}$} \\
\hline $\mathrm{mg} / \mathrm{dl}$ & $\mathrm{mmol} / \mathrm{l}$ & $\mathrm{mg} / \mathrm{dl}$ & $\Delta(\%)$ & $\mathrm{mmol} / \mathrm{l}$ & $\mathrm{mg} / \mathrm{dl}$ & $\Delta(\%)$ & $\mathrm{mmol} / \mathrm{l}$ & $\mathrm{mg} / \mathrm{dl}$ & $\Delta(\%)$ & $\mathrm{mmol} / \mathrm{l}$ \\
\hline 30,0 & 5,00 & 27,6 & $-8,0$ & 4,60 & 29,8 & $-0,7$ & 4,97 & 32,5 & $+8,3$ & 5,42 \\
\hline 50,0 & 8,33 & 46,0 & $-8,0$ & 7,67 & 48,6 & $-2,8$ & 8,10 & 49,1 & $-1,8$ & 8,18 \\
\hline 70,0 & 11,67 & 69,1 & $-1,3$ & 11,52 & 71,2 & $+1,7$ & 11,87 & 68,3 & $-2,4$ & 11,39 \\
\hline 90,0 & 15,00 & 91,4 & $+1,6$ & 15,24 & 93,2 & $+3,6$ & 15,54 & 89,3 & $-0,8$ & 14,89 \\
\hline 110,0 & 18,34 & 109,0 & $-0,9$ & 18,17 & 111,0 & $+0,9$ & 18,50 & 109,6 & $-0,4$ & 18,27 \\
\hline 150,0 & 25,00 & 146,0 & $-2,7$ & 24,34 & 148,4 & $-1,1$ & $24, \overline{74}$ & 151,6 & $+1,1$ & 25,27 \\
\hline 200,0 & 33,34 & 196,3 & $-1,9$ & 32,72 & 199,6 & $-0,2$ & 33,27 & 197,9 & $-1,1$ & 32,99 \\
\hline 300,0 & 50,01 & 298,3 & $-0,6$ & 49,72 & 300,7 & $+0,2$ & 50,12 & 302,7 & $+0,9$ & 50,46 \\
\hline
\end{tabular}
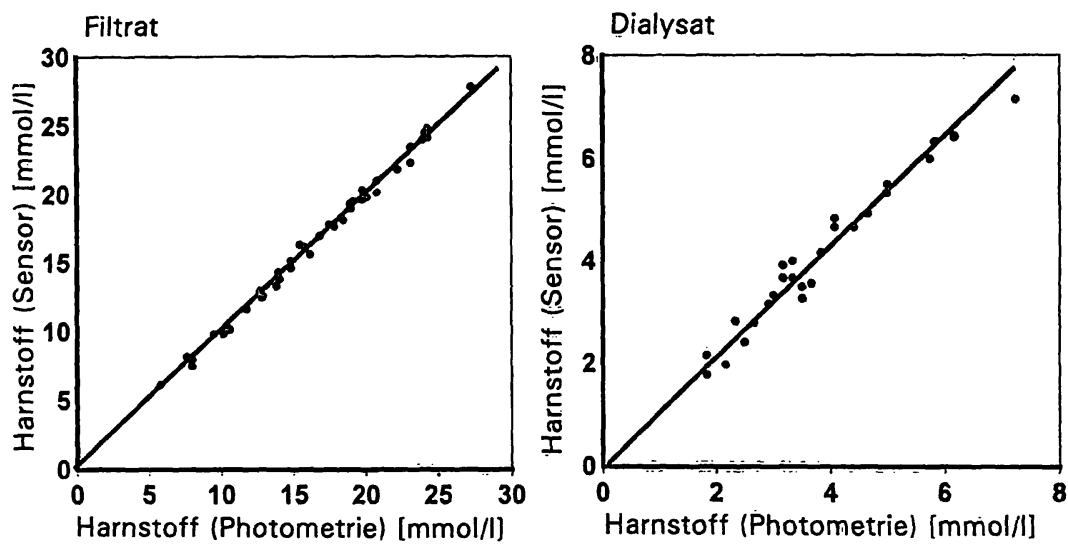

Abb. 3 Korrelation Harnstoff-Sensor/Photometrie

Filtrat: $r=0,997, p<0,001$; Dialysat: $r=0,983, p<0,001$

Tab. 3 Protoneninterferenz des Harnstoff-Sensors auf der Basis der Enzymmembran und der pH-abhängigen Gleichgewichtseinstellung zwischen $\mathrm{NH}_{4}^{+}$und $\mathrm{NH}_{3}$. Angegeben sind die vorgegebenen Harnstoffkonzentrationen (,Waage“), die mit dem Sensor ge- messenen Harnstoffkonzentrationen (mg/dl) und die Abweichungen gegenüber den vorgegebenen $(\Delta, \%)$ sowie die korrespondierenden Konzentrationen in mmol/l.

\begin{tabular}{|c|c|c|c|c|c|c|c|c|c|c|}
\hline \multirow{2}{*}{\multicolumn{2}{|c|}{ Harnstoff, Waage }} & \multicolumn{9}{|l|}{$\mathrm{H}^{+}$} \\
\hline & & \multicolumn{3}{|c|}{$\begin{array}{l}\mathrm{pH} 7,10 \\
\text { Harnstoff, Sensor }\end{array}$} & \multicolumn{3}{|c|}{$\begin{array}{l}\mathrm{pH} 7,40 \\
\text { Harnstoff, Sensor }\end{array}$} & \multicolumn{3}{|c|}{$\begin{array}{l}\text { pH } 7,70 \\
\text { Harnstoff, Sensor }\end{array}$} \\
\hline $\mathrm{mg} / \mathrm{dl}$ & $\mathrm{mmol} / \mathrm{l}$ & $\mathrm{mg} / \mathrm{dl}$ & $\Delta(\%)$ & $\mathrm{mmol} / \mathrm{l}$ & $\mathrm{mg} / \mathrm{dl}$ & $\Delta(\%)$ & $\mathrm{mmol} / \mathrm{l}$ & $\mathrm{mg} / \mathrm{dl}$ & $\Delta(\%)$ & $\mathrm{mmol} / \mathrm{l}$ \\
\hline 30,0 & 5,00 & 29,3 & $-2,3$ & 4,88 & 31,0 & $+3,3$ & 5,17 & 30,5 & $+1,7$ & 5,08 \\
\hline 50,0 & 8,33 & 50,7 & $+1,4$ & 8,45 & 51,6 & $+3,2$ & 8,60 & 48,5 & -3.0 & 8,08 \\
\hline 70,0 & 11,67 & 67,1 & $-4,1$ & 11,19 & 68,5 & $-2,1$ & 11,42 & 69,1 & $-1,3$ & 11,52 \\
\hline 90,0 & 15,00 & 87,8 & $-2,4$ & 14,64 & 90,6 & $+0,7$ & 15,10 & 91,5 & $+1,7$ & 15,25 \\
\hline 110,0 & 18,34 & 112,7 & $+2,5$ & 18,79 & 110,5 & $+0,5$ & 18,42 & 114,0 & $+3,6$ & 19,00 \\
\hline 150,0 & 25,00 & 152,0 & $+1,3$ & 25,34 & 147,5 & $-1,7$ & 24,59 & 148,9 & $-0,7$ & 24,82 \\
\hline 200,0 & 33,34 & 197,2 & $-1,4$ & 32,87 & 200,5 & $+0,3$ & 33,42 & 198,8 & $-0,6$ & 33,14 \\
\hline 300,0 & 50,01 & 296,3 & $-1,2$ & 49,39 & 303,8 & $+1,3$ & 50,64 & 298,7 & $-0,4$ & 49,79 \\
\hline
\end{tabular}

Die Urease erweist sich bei der enzymatischen Spaltung des Harnstoffs als hochselektiv. Suramin und Thioharnstoff hemmen sie kompetitiv (17). Die inhibitorische Wirkung von $\mathrm{Hg}^{2+}$ auf die Ureaseaktivität konnte unter
Einsatz einer gassensitiven Ammoniak-Elektrode zur Spurenbestimmung freier und komplexierter Quecksilberionen herangezogen werden (18). Inhibitorinterferenzen wurden für $\mathrm{Ag}^{+}$und $\mathrm{Cu}^{2+}$ beobachtet (18). Fluorid- 
Ionen sind im Meßgut unerwünscht, da sie die Urease reversibel nichtkompetitiv hemmen (19); dies ließ sich ebenfalls analytisch zur Messung von Fluoridionen mit einer durch immobilisierte Urease sensibilisierten $\mathrm{pCO}_{2-}$ Elektrode ausnutzen (20), wenngleich üblicherweise die hochselektive $\mathrm{LaF}_{3}$-Einkristall-Membranelektrode (21). zur Fluoridionenanalyse herangezogen wird.

Die geschwindigkeitsbestimmenden Schritte für die Einstellzeit der bioelektrochemischen Harnstoff-Elektrode sind die Diffusionsvorgänge in der hydrophilen Enzymmembran und die Kinetik der Urease; denn die Nonactin-PVC-Membran des ammoniumselektiven Festkontakt-Sensors hat eine Einstellzeit von $<1 \mathrm{~s}$. Eine Meßwertänderung von 40 auf $100 \mathrm{mg} / \mathrm{dl}(6,67$ auf 16,67 $\mathrm{mmol} / \mathrm{l}$ ) Harnstoff wird in $90 \mathrm{~s}$ angezeigt (Abb. 4). Die Steilheit des $\mathrm{NH}_{4}^{+}$-selektiven Detektors beträgt in reinen $\mathrm{NH}_{4} \mathrm{Cl}$-Lösungen $59,2 \mathrm{mV}$. Hingegen wurde bei einem 10 fachen Konzentrationsanstieg des Harnstoffs in $\mathrm{Ge}-$ genwart der im Blut vorkommenden Störionenkonzentrationen eine Differenz in der elektromotorischen Kraft der elektrochemisch-enzymatischen Meßkette von 42,6 $\mathrm{mV}$ gefunden. Ein Wechsel der Enzymmembran wird bei einem Aktivitätsverlust der Urease von $20 \%$ vorgenommen. Dem entspricht gegenwärtig bei kontinuierlichem Durchflußbetrieb eine Funktionsdauer von durchschnittlich vier Wochen. In Tabelle 4 sind zwei Harnstoff-Sensoren hinsichtlich ihrer Präzision in der Serie und von Tag zu Tag vergleichend gegenübergestellt.

Die sehr gute Korrelation der mittels Harnstoff-Sensor und Photometer bestimmten Harnstoffkonzentrationen im Filtrat und Dialysat geht aus Abbildung 3 hervor. Da die bioelektrochemisch erfaßten Filtrat-Harnstoffkonzentrationen ausgezeichnet mit den photometrisch im Blut ermittelten korrelieren $(r=0,99 ; p<0,001)$, darf die kontinuierliche Registrierung im Filtrat mit der Harnstoff-Membranelektrọde als repräsentativ für das Blut gelten.
Das Ergebnis einer mit zwei bioelektrochemischen Harnstoff-Elektroden durchgeführten Prozeßkontrolle des Behandlungsvorganges mit der Künstlichen Niere ist in Abbildung 5 zusammenfassend dargestellt. In Abbildung 5 oben läßt sich die Wirksamkeit der Hämodialysebehandlung an der Abnahme der Harnstoff-Konzentration im Filtrat des Blutes als Äquivalent zur aktuellen Blutharnstoffkonzentration kontinuierlich verfolgen, parallel dazu zeigt Abbildung 5 Mitte den Verlauf im abfließenden Dialysat. Unter Berücksichtigung des Dialysatflusses kann aus beiden Konzentrationen die eliminierte Harnstoffmenge gemäß Abbildung 5 unten exakt berechnet werden.

\section{Diskussion}

Auf die Bedeutung der bioelektrochemischen Prozeßkontrolle der Dialysebehandlung haben wir eingangs hingewiesen. Unterschiedliche Verfahrensweisen zur elektrochemisch-enzymatischen Analyse des Harnstoffs wurden über viele Jahre weiterentwickelt (9). Der Nachweis für die Praktikabilität einer potentiometrischen Harnstoffmessung läßt sich mit Hilfe einer einfachen stationären Versuchsanordnung erbringen, wenn einer thermostatisierten, gerührten Harnstofflösung Urease zugesetzt und aus der dann meßioninduzierten Potentialänderung auf die Substratkonzentration geschlossen wird (22).

G. G. Guilbault und J. G. Montalvo Jr: beschrieben 1969 eine Enzymelektrode zur Messung des Harnstoffs auf der Basis einer auf Ammoniumionen ansprechenden Glasmembranelektrode und aufpolymerisiertem Acrylamid-Gel mit immobilisierter Urease (23). Durch zusätzliche Applikation einer Zellophan-Folie zwecks Verhinderung von Auswascheffekten der immobilisierten Urease aus der photopolymerisierten Acrylamidgel-Matrix wurde eine Verbesserung erzielt (24). Die Detektion der freigesetzten Ammoniumionen über eine ammoni-

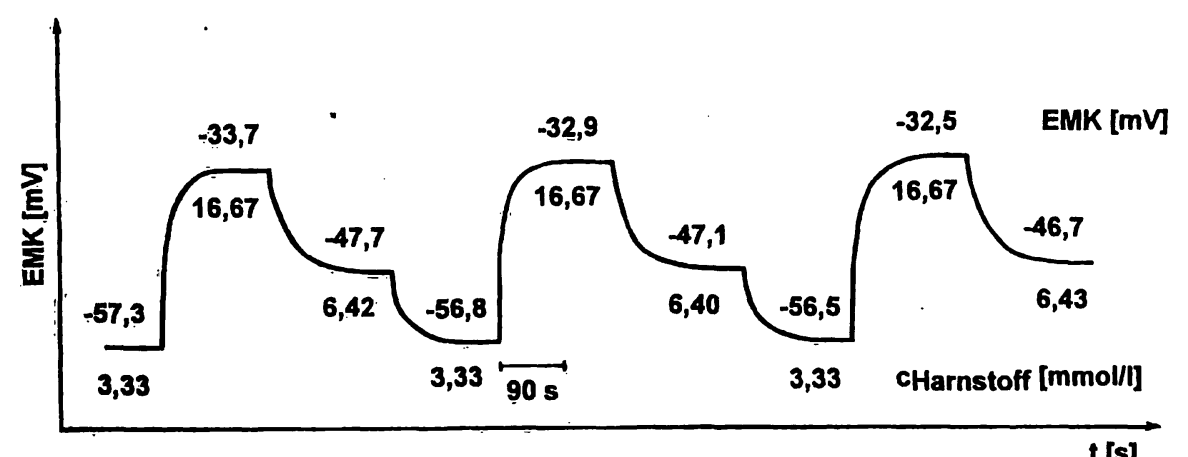

Abb. 4 Harnstoff-Meßkurve mit drei Analysen in wäßrigen, hinsichtlich der Kationenkonzentrationen hämoanalogen Elektrolytlösungen und jeweiliger Zweipunkt-Kalibrierung. (Harnstoff 20;0 und $100,0 \mathrm{mg} / \mathrm{dl}$ bzw. 3,33 und 16,67 mmol/l). Den unterhalb der Meßkurve angegebenen Harnstoffkonzentrationen zuzuordnende EMK-Werte sind jeweils oberhalb der Meßkurve aufgefulurt. 
Tab. 4 Vergleich zweier Harnstoff-Sensoren. Präzision in der Seric (Repetierbarkeit) und von Tag zu Tag (Reproduzierbarkeit). Soll-Wert: $40,0 \mathrm{mg} / \mathrm{dl}(6,67 \mathrm{mmol} / \mathrm{l})$ Harnstoff. Angegeben sind
Meßwerte sowie Qualitätsmerkmale der Harnstoffkonzentrationen in $\mathrm{mg} / \mathrm{dl}$ und korrespondierend in $\mathrm{mmol} / \mathrm{l}$ sowie die Variationskoeffizienten in \%.

\begin{tabular}{|c|c|c|c|c|c|c|c|c|}
\hline \multirow{3}{*}{$\begin{array}{l}\text { Harnstoff- } \\
\text { Messung }\end{array}$} & \multicolumn{4}{|l|}{ Sensor I } & \multicolumn{4}{|l|}{ Sensor II } \\
\hline & \multicolumn{2}{|l|}{$\operatorname{Tag} A$} & \multicolumn{2}{|l|}{ Tag B } & \multicolumn{2}{|l|}{ Tag A } & \multicolumn{2}{|l|}{ Tag B } \\
\hline & $\mathrm{mg} / \mathrm{dl}$ & $\mathrm{mmol}$ & $\mathrm{mg} / \mathrm{dl}$ & $\mathrm{mmol} / \mathrm{l}$ & $\mathrm{mg} / \mathrm{dl}$ & $\mathrm{mmol} / \mathrm{l}$ & $\mathrm{mg} / \mathrm{dl}$ & $\mathrm{mmol} / \mathrm{l}$ \\
\hline $\begin{array}{l}1 . \\
2 . \\
3 . \\
4 .\end{array}$ & $\begin{array}{l}38,2 \\
37,8 \\
37,8 \\
38,2\end{array}$ & $\begin{array}{l}6,37 \\
6,30 \\
6,30 \\
6,37\end{array}$ & $\begin{array}{l}40,3 \\
38,3 \\
38,4 \\
37,4\end{array}$ & $\begin{array}{l}6,72 \\
6,38 \\
6,40 \\
6,23\end{array}$ & $\begin{array}{l}38,4 \\
38,6 \\
38,5 \\
38,5\end{array}$ & $\begin{array}{l}6,40 \\
6,43 \\
6,42 \\
6,42\end{array}$ & $\begin{array}{l}38,0 \\
37,9 \\
38,4 \\
37,9\end{array}$ & $\begin{array}{l}6,33 \\
6,33 \\
6,40 \\
6,32\end{array}$ \\
\hline Mittelwert & 38,0 & 6,33 & 38,6 & 6,43 & 38,5 & 6,42 & 38,1 & 6,34 \\
\hline $\begin{array}{l}\text { Standard- } \\
\text { abweichung } s_{\mathrm{R}}\end{array}$ & 0,23 & 0,040 & 1,22 & 0,206 & 0,08 & 0,013 & 0,24 & 0,039 \\
\hline $\begin{array}{l}\text { Variations- } \\
\text { koeffizient VK }\end{array}$ & \multicolumn{2}{|c|}{$0,61 \%$} & \multicolumn{2}{|c|}{$3,16 \%$} & \multicolumn{2}{|c|}{$0,21 \%$} & \multicolumn{2}{|c|}{$0,63 \%$} \\
\hline $\begin{array}{l}\text { Mittlerer Fehler } \\
\text { des Mittelwertes }\end{array}$ & 0,12 & 0,020 & 0,61 & 0,103 & 0,04 & 0,006 & 0,12 & 0,019 \\
\hline $\begin{array}{l}95 \% \text {-Ver- } \\
\text { trauensbereich }\end{array}$ & $37,8-38,2$ & $6,29-6,37$ & $37,4-39,8$ & $6,22-6,64$ & $38,4-38,6$ & $6,41-6,43$ & $37,9-38,3$ & $6,30-6,38$ \\
\hline
\end{tabular}
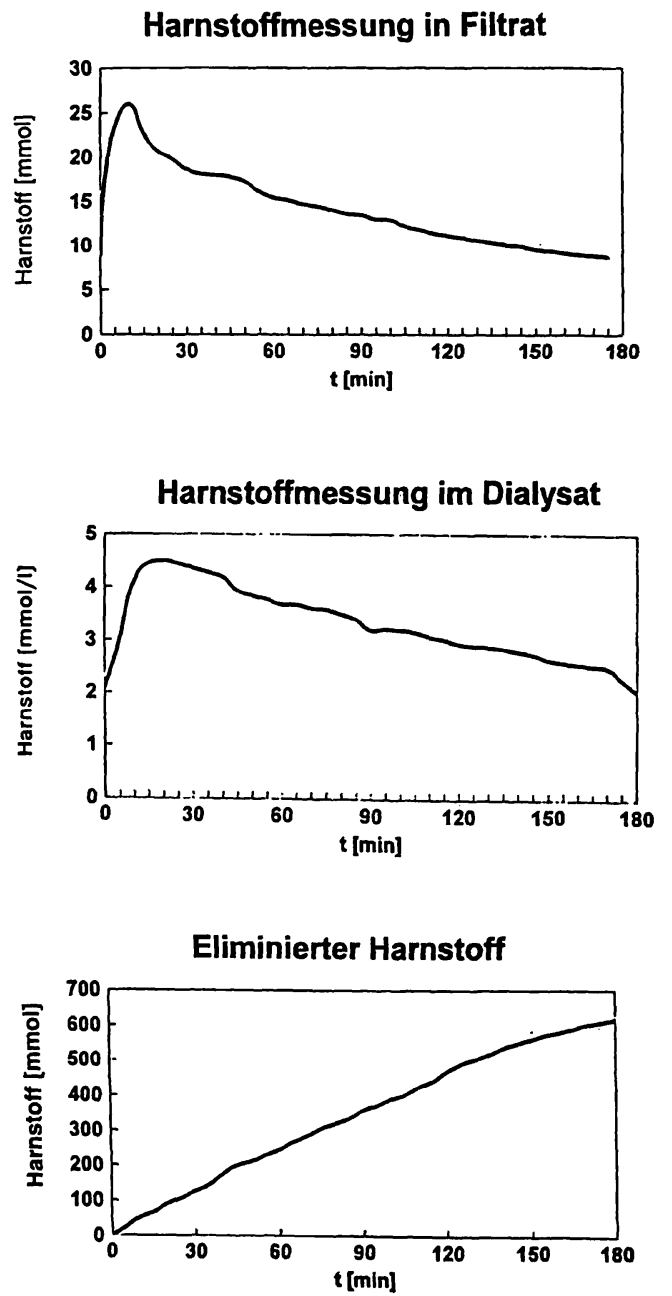

Abb. 5 Oben: Kontinuierlicher Verlauf im Filtrat des Blutes (Harnstoff-Sensor I in Abb. 2), Mitte: im abfließenden Dialysat (Harnstoff-Sensor II in Abb. 2) und schließlich unten: die daraus unter gleichzeitiger Volumenmessung errechnete Menge des eliminierten Harnstoffs während einer Hämodialyse. umsensitive, beziehungsweise genauer als kationenselektiv zu bezeichnenden Glasmembranelektrode weist eine sehr hohe Querempfindlichkeit gegenüber anderen Kationen wie $\mathrm{K}^{+}$und sogar $\mathrm{Na}^{+}$auf (25). Die diesbezüglich kompensatorische zusätz̄liche Verwendung einer unbehandelten Kationen-Vergleichselektrode in einem Ionenaustausch-System (26) stellt für die hier zur Diskussion stehende Applikation einen unverhältnismäßig hohen, technisch nicht vertretbaren Aufwand dar und birgt eine Reihe von Unsicherheitsfaktoren. Mit dem Ersatz der ammoniumsensitiven Glasmembranen des Detektors durch Nonactin-Silikongummi-Membranen wurde eine genaue Messung bei bekanntem Kaliumgehalt in biologischen Flüssigkeiten möglich (27), wobei Harnstoffanalysen im Serum auch weiterhin zusätžlich $\mathrm{Maßnahmen} \mathrm{wie} \mathrm{eine} \mathrm{kontrollierte} \mathrm{Verdünnung} \mathrm{auf} \mathrm{ei-}$ nen konstanten Interferenz-Spiegel im Zusammenhang mit einem Drei-Elektroden-System verlangten (28). Es ist daher nicht verwunderlich, wenn die Entwicklung von Harnstoff-Sensoren auch unter Anwendung anderer elektrochemischer Detektoren wie für pH (29), $\mathrm{CO}_{2}$ (30) oder $\mathrm{NH}_{3}$ (31) weiter betrieben wurde und bis in die Gegenwart $(7,8,32,33)$ ein aktuelles Interessè an der Entwicklung von Harnstoff-Sensoren existiert. Auch über Katheter-Elektroden wurde früher bereits berichtet $(9,34)$. Obwohl polare neutrale Moleküle wie Harnstoff und Thioharnstoff durch acyclische kronenetherartige Neutralliganden komplexiert werden (35), ist gegenwärtig der Weg über eine harnstoffselektive Carrier-PVCMembranelektrode nicht realisierbar.

Die Verfahrensweise, den Harnstoff in kontinuierlichem Durchflußverfahren unter fơtlaufendem Zupumpen lös- 
licher Urease zu spalten und die dabei freigesetzten Ammoniumionen mit einer Nonactin-Disk-Elektrode (36) zu detektieren, haben wir bereits 1977/78 beschrieben (37), die nun zur Entwicklung des hier im Detail vorgestellten Harnstoff-Sensors geführt hat. Über seinen ErstEinsatz zur Prozeßkontrolle an der Künstlichen Niere wurde von uns kürzlich berichtet (8). Abgesehen von der wirtschaftlicheren Form der Elektroanalyse mit membranständig immobilisierter Urease liegt der tech- nologische Fortschritt des Harnstoff-Biosensors in der kompakteren Bauform dieses bedside-analysers und damit wesentlich praktikableren Applikationsform sowie schnelleren Verfügbarkeit der Meßdaten. Darüberhinaus besteht kein Bedarf mehr für eine kaliumselektive Korrekturmessung. Ferner gestattet der Harnstoff-Durchflußsensor wegen seines sauerstoffunabhängigen Festkontaktes auch Direktmessungen im heparinisierten Blut.

\section{Literatur}

1. Schindler, J. G., Schindler, M. M., Herna, K., Reisinger, E., Kuhlmann, U., Gräf, R. \& Lange, H. (1991) Ionenselektiver Elektroanalysator mit tubulären Festkontakt-Durchflußsensoren für die kontinuierlich bioelektrochemisch kontrollierte Hämodialyse von $\mathrm{K}^{+}, \mathrm{Na}^{+}, \mathrm{Ca}^{2+}, \mathrm{Cl}^{-}$und $\mathrm{pH}$. Biomed. Technik $36,271-284$.

2. Kuhlmann, U., Gräf, R., Schindler, J. G. \& Lange, H. (1992) Continuous ionography (CIG) in haemodialysis by ion-selective carrier membrane electrodes (ISCME) with solid cement contact for flow-through measurement. The International Journal of Artificial Organs 15, 209-212.

3. Kuhlmann, U., Gräf, R., Schindler, J. G. \& Lange, H. (1992) Ionensensitive Elektroden als Sensoren für die computermodulierte Hämodialyse. Klin. Wochenschr. 109, KLWOAZ 69, Suppl. XXVIII, S. 35 P 78.

4. Lindsay, R. M., Heidenheim, A. P., Spanner, E., Baird, J., Simpson, K. \& Allison, M. E. (1991) Die künftige Entwicklung. Realzeitliche Harnstoff-Überwachung während jeder Dialyse sichert die Qualität und Rentabilität. Journal für das Nephrologische Team 3, 4-8.

5. Harter, H. R. (1983) Review of significant findings from the National Cooperative Dialysis Study and recommendations. Kidney International 23, Suppl. 13, 107-112.

6. Lowrie, E. G., Laird, N. M. \& Henry, R. R. (1983) Protocol for the National Cooperative Dialysis Study. Kidney International 23, Suppl. 13, 11-18.

7. Schindler, J. G., Schindler, M. M. \& Herna, K. (1992) Molekularselektiver Harnstoff-Biosensor. In: High-Tech: Bio- und Chemosensoren (Schindler, J. G., ed.) Informationsschrift der Philipps-Universität Marburg. 3. erweiterte Auflage zur Analytica 92 in München vom 5.-8. Mai.

8. Schindler, J. G., Schindler, M. M., Herna, K., Kuhlmann, U., Knaack, J. \& Lange, H. (1993) Harnstoff-Biosensor zur Prozeßkontrolle an der künstlichen Niere. Biomed. Technik 38, 118-119.

9. Schindler, J. G. \& Schindler, M. M. (1983) Bioelektrochemische Membranelektroden. Walter de Gruyter, Berlin, New York.

10. Neupert-Laves, K. \& Dobler, M. (1976) The crystal structure of the $\mathrm{NH}_{4} \mathrm{NCS}$ complex of nonactin. Helv. Chim. Acta 59, 614-623.

11. Dobler, M., Dunitz, J. D. \& Kilbourn, B. T. (1969) Die Struktur des KNCS-Komplexes von Nonactin. Helv. Chim. Acta 52, 2573-2583.

12. Dobler, M. \& Phizackerley, R. P. (1974) The Crystal Structure of the NaNCS Complex of Nonactin. Helv. Chim. Acta 57, 664-674.

13. Guilbault, G. G., Durst, R. A., Frant, M. S., Freiser, H., Hansen, E. H., Light, T. S., Pungor, E., Rechnitz, G., Rice, N. M., Rohm, T. J., Simon, W. \& Thomas, J. D. R. (1976) Recommendations for nomenclature of ion-selective electrodes. Pure Appl. Chem. 48, 127-132.

14. Kuhlmann, U., Knaack, J., Schindler, J. G., Schindler, M. M., Herna, K., Schmidt, B. \& Lange, H. (im Druck) Continuous "on line" monitoring of urea concentration in blood and dialysis fluid by molecular selective electrodes in haemodialysis. Nephrology Dialysis Transplantation.

15. Kuhlmann, U., Knaack, J., Schindler, J. G., Schindler, M. M., Herna, K., Schmidt, B. \& Lange, H. (1993) Continuous "on line" monitoring of urea concentration in blood and dialysis fluid by molecular selective electrodes in haemodialysis. Vortrag auf dem XXXth Congress of the EDTA European Renal Association, 15.-18. Sept., Glasgow, Scotland.

16. Kassenärztliche Vereinigung Hessen (1989) Qualitätssicherung im Labor - Neue Richtlinien der Bundesärztekammer. Hessisches Ärzteblatt 7, 428.

17. Barman, T. E. (1969) Enzyme Handbook. Springer-Verlag, Berlin, Bd. 2, S. 648 zit. nach Bergmeyer, H. U. (1974) Methoden der enzymatischen Analyse. Verlag Chemie Weinheim/ Bergstr. Bd. I, 3. Aufl., pp. 552-553.

18. Ögren, L. \& Johansson, G. (1978) Determination of traces of mercury (II) by inhibition of an enzyme reactor electrode loaded with immobilized urease. Anal. Chim. Acta 96, 1-11.

19. Cimasoni, G. (1969) Fluor et enzymes. Rev. Mens. Suisse Odonto-Stomatol. 79, 911-926.

20. Tran-Minh, C. \& Beaux, J. (1979) Enzyme electrode for inhibitors determination: Urease-fluoride system. Anal. Chem. $51,91-95$.

21. Frant, M. S. \& Ross, J. W., Jr. (1966) Electrode for sensing fluoride ion activity in solution. Science $154,1553-1555$.

22. Katz, S. A. \& Rechnitz, G. A. (1963) Direct potentiometric determination of urea after urease hydrolysis. Z. Anal. Chem. 196, 248-251.

23. Guilbault, G. G. \& Montalvo, J. G. Jr. (1969) A urea-specific enzyme electrode. J. Amer. Chem. Soc. 91, 2164-2165.

24. Guilbault, G. G. \& Montalvo, J. G., Jr. (1969) An improved urea specific enzyme electrode. Anal. Lett. 2, 283-293.

25. Guilbault, G. G. \& Montalvo, J. G. (1970) An enzyme electrode for the substrate urea. J. Amer. Chem. Soc. 92, 25332538.

26. Guilbault, G. G. \& Hrabankova, E. (1970) Determination of urea in blood and urine with a urea-sensitive electrode. Anal. Chim. Acta 52, 287-294.

27. Guilbault, G. G. \& Nagy, G. (1973) Improved urea electrode. Anal. Chem. 45, 417-419.

28. Guilbault, G. G., Nagy, G. \& Kuan, S. S. (1973) An improved electrode for the assay of urea in blood. Anal. Chim. Acta 67, 195-201.

29. Nilsson, H., Akerlund, A.-C. \& Mosbach, K. (1973) Determination of glucose, urea and penicillin using enzyme-pH-electrodes. Biochim. Biophys. Acta 320, 529-534.

30. Guilbault, G. G. \& Shu, F. R. (1972) Enzyme electrodes based on the use of a carbon dioxide sensor. Urea and L-tyrosine electrodes. Anal. Chem. 44, 2161-2166.

31. Hansen, E. H. \& Ruzicka, J. (1974) Enzymatic analysis by means of the air-gap electrode determination of urea in blood. Anal. Chim. Acta 72, 353-364.

32. Przybyt, M. \& Sugier, H. (1990) Tungsten electrode for urea. Anal. Chim. Acta 239, 269-276. 
33. Narinesingh, D., Mungal, R. \& Ngo, T. T. (1991) Urease coupled to poly(vinyl alcohol) activated by 2-fluoro-1-methylpyridinium salt: Preparation of a urease potentiometric electrode and application to the determination of urea in serum. Anal. Chim. Acta 249, 387-393.

34. Schindler, J. G. \& v. Gülich, M. (1981) Technologien für elektrochemische Festkontakt-Katheter-Sensoren und TeleskopKatheter-Elektroden-Systeme zur Bestimmung von $\mathrm{Ca}^{2+}, \mathrm{K}^{+}$, $\mathrm{Na}^{+}, \mathrm{Cl}^{-}$, Harnstoff, $\mathrm{pH}, \mathrm{O}_{2}$ und $\mathrm{CO}_{2}$. Biomed. Technik 26, 43-53.

35. Raßhofer, W. \& Vögtle, F. (1978) Complexes of noncyclic crown-type polyethers with thiourea and urea. Tetrahedron Lett. 3, 309-312.

36. Schindler, J. G., Schindler, R. G. \& Aziz, O. (1978) Ammoniumselektive Disk-Elektrode mit dem Carrier-Antibiotikum Nonactin. Verfahren zur kontinuierlichen enzymatischen Elektro- analyse von Harnstoff, I. Mitteilung. J. Clin. Chem. Clin. Biochem. 16, 441-445.

37. Schindler, J. G., Schindler, R. G. \& Aziz, O. (1978) Ein $\mathrm{NH}_{4}^{+}$-selektiv-enzymatisches Durchflußsystem. Verfahren zur kontinuierlichen enzymatischen Elektroanalyse von Harnstoff, II. Mitteilung. J. Clin. Chem. Clin. Biochem. 16, 447-450.

Prof. Dr. Dr. J. G. Schindler

Institut für Normale und

Pathologische Physiologie

Projekt Biomedizinische Technik und

Bioelektrochemische Membranelektrodèn

Philipps-Universität

Karl-von-Frisch-Straße

D-35033 Marburg/Lahn

Germany 